\title{
Enraizamento de estacas semilenhosas de cultivares de oliveira
}

\author{
Luiz Fernando de Oliveira da Silva (1); Adelson Francisco de Oliveira (2); Rafael Pio ( $\left.{ }^{\text {* }}\right)$; Carolina \\ Ruiz Zambon ('); Dili Luiza Oliveira ( $\left.{ }^{2}\right)$ \\ (') Departamento de Agricultura, Universidade Federal de Lavras (UFLA), Caixa Postal 3037, 372000-000 Lavras (MG), Brasil. \\ (2) Empresa de Pesquisa Agropecuária de Minas Gerais (EPAMIG-CTSM), Caixa Postal 176, 37200-000 Lavras (MG), Brasil. \\ (*) Autor correspondente: rafaelpio@dag.ufla.br
}

Recebido: 18/maio/2012; Aceito: 19/dez./2012

\section{Resumo}

O objetivo deste trabalho foi avaliar o enraizamento de estacas semilenhosas provenientes de 35 cultivares de oliveira, coletadas em duas épocas do ano (abril e agosto). O delineamento experimental utilizado foi o inteiramente casualizado, em esquema fatorial 35×2, com quatro repetições e 25 estacas por parcela. As estacas foram preparadas com aproximadamente $12 \mathrm{~cm}$ de comprimento e 4 a 6 internódios, com quatro folhas inteiras na região apical. 0 tratamento com solução

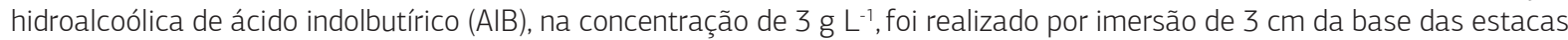
na solução, por cinco segundos. As estacas foram acondicionadas em bancadas preenchidas com areia e colocadas em casa de vegetação com nebulização intermitente. Decorridos 70 dias, verificou-se que o potencial de enraizamento das estacas é de grande variação entre as cultivares de oliveira. As cultivares MGS MANZ215 e MGS TAF390 se destacaram pela maior capacidade de enraizamento. A estaquia em abril favorece o enraizamento, em relação à estaquia realizada em agosto, para algumas das cultivares avaliadas.

Palavras-chave: Olea europaea L., propagação, estaquia.

\section{Rooting of semi-woody cuttings of olive cultivars}

\begin{abstract}
The aim of this study was to evaluate the semi-woody rooting of cuttings from 35 olive cultivars collected at two different times (April and August). The experimental design was the completely randomized in factorial 35x2, with 4 replicates and 25 cuttings per plot. The cuttings were standardized to $12 \mathrm{~cm}$ long and 4-6 internodes, with four entire leaves in the apical region. The treatment with hydroalcoholic solution of indolebutyric acid (IBA) at concentration of $3 \mathrm{~g} \mathrm{~L}^{-1}$ was performed by immersion of $3 \mathrm{~cm}$ from the base of the cuttings in the solution, for five seconds. The cutting was planted in sand and placed in greenhouse with intermittent mist. After 70 days, there was a significant variation in rooting among the olive cultivars. The cultivars MGS MANZ215 and MGS TAF390 showed higher rooting capacity. The cuttings collected in April presented higher rooting as compared to those ones collected in August for some of the cultivars evaluated.
\end{abstract}

Key words: Olea europaea L., propagation, cuttings.

O cultivo de oliveiras no Brasil é uma atividade agrícola recente e em expansão (Oliveira et al., 2009). Nos últimos nove anos, o aumento do consumo de azeite de oliva e azeitona no Brasil foi de $120 \%$ e $45 \%$ respectivamente. Este aumento deve-se a fatores como: divulgaçáo dos benefícios da dieta mediterrânea na saúde; entrada de produtos no mercado interno com preços mais acessíveis e aumento do poder aquisitivo de algumas classes sociais (BRASIL, 2012).

Atualmente, na Serra da Mantiqueira existem aproximadamente 300 mil plantas distribuídas entre 60 produtores e 55 municípios. Entretanto, para suprir o mercado interno (214 mil toneladas) é necessário o plantio de 11 milhóes de plantas, exigindo da pesquisa informaçóes técnicas para sua produção (Oliveira et al., 2012; Silva et al., 2012).

Apesar de o fruto da oliveira possuir semente viável, a reprodução sexual não é desejada no estabelecimento de plantios comerciais, em razão da segregaçáo genética, que origina indivíduos com características distintas da planta-matriz, e em razão do longo período juvenil, caracterizado por, pelo menos, oito anos (Oliveira et al., 2009); a propagação vegetativa é a alternativa viável de produção de mudas para manter as características genéticas das cultivares (VIlLA et al., 2003; SMARSI et al., 2008).

A estaquia é a propagação vegetativa mais utilizada para a oliveira em todos os países produtores, com enraizamento médio de estacas semilenhosas em torno 
de 54,5\% (Oliveira et al., 2010a). Nesse contexto, para maximizar o enraizamento das estacas, foi realizada uma série de trabalhos no Brasil, sendo definido que estacas semilenhosas dotadas de quatro folhas e tratadas com ácido indolbutírico na concentração de $3 \mathrm{~g} \mathrm{~L}^{-1}$ propicia aumentos na emissão de raízes, com resultados variando de 25,5\% a 61,24\% (Pio et al., 2005; Oliveira et al., 2009; Oliveira et al., 2010a,b).

Porém, tem-se observado influência da época de coleta das estacas de oliveira no enraizamento. Em trabalhos desenvolvidos com a cultivar Ascolano 315 (MGS ASC315), no Sul de Minas Gerais, verificou-se que as estacas coletadas em abril atingiram 20,8\% de enraizamento e emissão de 6,2 raízes (Oliveira et al., 2003). Quando coletadas em agosto, o enraizamento foi reduzido para 7,3\% e ocorreu emissão de 2,5 raízes (Oliveira et al., 2009). Para a estaquia em setembro, verificaram-se $15,8 \%$ de estacas enraizadas e emissão de 2,5 raízes (Oliveira et al., 2010a), e em novembro, o enraizamento foi de $8,8 \%$, com apenas 1,3 raízes por estaca (Oliveira et al., 2010b).

Assim, verifica-se que a época de coleta das estacas e estaqueamento influencia a formação de raízes adventícias, sendo importante a definiçâao do período ideal de coleta desse material.

Assim, um ponto importante a ser definido, quando da coleta das estacas, é o conhecimento das fenofases das oliveiras cultivadas no Sul de Minas, pois determinadas fases do ciclo da cultura influencia no processo de enraizamento, em função da concentração de reserva acumulada, o que interfere na relação $\mathrm{C} / \mathrm{N}$.

Outro fator a ser observadoéo potencial genético das cultivares de oliveira em emitirem raízes. Del Río e Caballero (2005) constataram diferenças ao comparar 152 cultivares de oliveiras do Banco de Germoplasma de Córdoba, Espanha.

Com isso, torna-se necessário o conhecimento do potencial rizogênico das cultivares de oliveiras cultivadas no Sul de Minas, uma vez que, dentro do programa de melhoramento genético desta cultura visa-se à seleção de acessos superiores adaptáveis às condiçōes edafoclimáticas locais e uma vez selecionados deve-se, posteriormente, lançá-los como cultivar comercial.

Diante do exposto, o objetivo deste trabalho foi avaliar o enraizamento de estacas provenientes de 35 cultivares de oliveira, colhidas em duas épocas do ano (abril e agosto).

As oliveiras utilizadas neste trabalho pertencem ao Banco Ativo de Germoplasma (BAG) situado na Fazenda Experimental da EPAMIG, em Maria da Fé, Sul de Minas Gerais, a $22^{\circ} 18^{\prime}$ de latitude Sul e $45^{\circ} 23^{\prime}$ de longitude Oeste, altitude média de 1.276 metros e classificação climática de Köppen tipo Cwb, ou seja, clima temperado chuvoso (mesotérmico), também denominado subtropical de altitude e solo do tipo Latossolo Vermelho com textura média argilosa.

O pomar foi instalado em 2005 com plantas obtidas a partir de estacas enraizadas, sendo seis plantas de cada cultivar, com espaçamento de 7x6. Foram coletadas estacas semilenhosas da regiáo mediana de plantas de 35 cultivares, em abril e agosto de 2010. As cultivares avaliadas foram: Alto D'Ouro, Arbequina, MGS ASC315, MGS ASC322, MGS ASC323, Ascolano USA, Cerignola, MGS ZAL010, MGS SEVERO, MGS VIGLIONE, MGS GRAP084, MGS NEBLINA, Cornicabra, Empeltre, Galega, MGS GRAP541, MGS GRAP550, MGS GRAP553, MGS GRAP556, MGS GRAP561, MGS GRAP575, JB 1, MGS MANZ215, MGS MANZ234, MGS MARIENSE, Mission, MGS MISS293, Negroa, Penafiel SP, Picual, MGS ROP398, MGS SAL488, Santa Catalina, MGS TAF390 e MGS TAF391.

O delineamento experimental utilizado foi inteiramente casualisado, com tratamentos arranjados no esquema fatorial $35 \times 2$ (trinta e cinco cultivares e duas épocas de coleta), com quatro repetiçóes e 25 estacas por parcela.

As estacas foram preparadas com aproximadamente $12 \mathrm{~cm}$ de comprimento e 4 a 6 internódios, com quatro folhas inteiras na regiáo apical. $\mathrm{O}$ tratamento com solução hidroalcoólica de ácido indolbutírico (AIB), na concentração de $3 \mathrm{~g} \mathrm{~L}^{-1}$, foi realizado por imersão de $3 \mathrm{~cm} \mathrm{da}$ base das estacas na solução, por cinco segundos, conforme recomendaçóes de Oliveira et al. (2009).

A estaquia foi realizada na casa de vegetação com nebulização intermitente automatizada, acionada das 7 às 19 horas, em intervalos de 10 minutos, por 10 segundos, mantendo a umidade relativa em torno de $80 \%$ e temperatura entre 23 e $27{ }^{\circ} \mathrm{C}$ em seu interior. As estacas foram colocadas para enraizar em bancadas preenchidas com areia de textura média, com dimensões de $1 \mathrm{~m}$ de largura, $5 \mathrm{~m}$ de comprimento e $0,25 \mathrm{~m}$ de profundidade, no espaçamento de $3 \mathrm{~cm}$ na linha e $4 \mathrm{~cm}$ entre linhas, sendo cada linha ocupada por uma cultivar. Decorridos 70 dias, foram avaliados a porcentagem de estacas enraizadas e com calos, o número e o comprimento médio de raízes por estaca.

As variáveis em porcentagem foram transformadas por arco seno da raiz de $\mathrm{x} / 100$ e o número e comprimento médio de raízes em $(\mathrm{x}+0,5)^{1 / 2}$, com a finalidade de proporcionar a normalidade dos dados. Os resultados foram submetidos à análise de variância e as médias agrupadas pelo teste de Scott-Knott, a 5\% de probabilidade de erro, utilizando o software estatístico Sisvar ${ }^{\mathbb{B}}$ (FERreira, 2008).

Houve efeito significativo da interação cultivares $\mathrm{x}$ épocas de estaquia para a porcentagem de estacas enraizadas e com calos e número médio de raízes por estaca. Para o comprimento médio das raízes por estaca, houve diferença entre os tratamentos, mas sem interaçáo com as épocas de coleta de estacas.

Observou-se que em de abril, as cultivares MGS MANZ215 e MGS TAF390 foram enquadradas no grupo com a maior porcentagem de enraizamento, obtendo-se média de 73\% (Tabela 1). Para estacas coletadas em agosto, o grupo com maior porcentagem 
de enraizamento proporcionou média de $45 \%$ e $57 \%$, sendo as cultivares MGS ASC322 e MGS TAF390 respectivamente.

A oliveira MGS TAF390 destacou-se entre as cultivares avaliadas com maior capacidade de enraizamento, independentemente da época de coleta das estacas.

O estádio fenológico e as condiçōes ambientais alteram o conteúdo de cofatores e acúmulo de inibidores do enraizamento. Alguns trabalhos relataram, também, que o fluxo e a mobilizaçáo das substâncias de reservas, principalmente carboidratos, influenciam a emissão das raízes (Pio et al., 2007; Daneluz et al., 2009; Han et al., 2009; Ohland et al., 2009). Nesse caso, a mobilização dos carboidratos na planta matriz está relacionada com o estádio

Tabela 1. Porcentagem de estacas semilenhosas enraizadas e com calo, coletadas de diferentes cultivares de oliveira em duas épocas do ano (abril e agosto)

\begin{tabular}{|c|c|c|c|c|c|}
\hline \multirow{3}{*}{$\begin{array}{l}\text { Cultivar } \\
\text { Alto D'Ouro }\end{array}$} & \multicolumn{3}{|c|}{ Estacas enraizadas (\%) } & \multicolumn{2}{|c|}{ Estacas com calo (\%) } \\
\hline & abril & \multicolumn{2}{|c|}{ agosto } & abril & agosto \\
\hline & $5 \mathrm{cA}$ & 0 & $c A$ & 64 bA & 73 aA \\
\hline Arbequina & $26 \mathrm{bA}$ & 24 & bA & $32 \mathrm{cA}$ & $37 \mathrm{CA}$ \\
\hline MGS ASC315 & 39 bA & 19 & bB & $34 \mathrm{cA}$ & $44 \mathrm{cA}$ \\
\hline MGS ASC322 & $44 \mathrm{bA}$ & 57 & $\mathrm{aA}$ & $26 \mathrm{cA}$ & $34 \mathrm{cA}$ \\
\hline MGS ASC323 & $12 \mathrm{cA}$ & 0 & cA & $81 \mathrm{aA}$ & 67 bA \\
\hline Ascolano USA & $28 \mathrm{bA}$ & 27 & bA & $30 \mathrm{cA}$ & $37 \mathrm{cA}$ \\
\hline Cerignola & $40 \mathrm{bA}$ & 11 & $c B$ & $18 \mathrm{dA}$ & $26 \mathrm{cA}$ \\
\hline MGS ZAL010 & 34 bA & 11 & $\mathrm{cB}$ & $38 \mathrm{cA}$ & 52 bA \\
\hline MGS SEVERO & 34 bA & 4 & $c B$ & $37 \mathrm{cA}$ & $6 \mathrm{~dB}$ \\
\hline MGS VIVLIONE & $4 \mathrm{cA}$ & 0 & $c A$ & 82 aA & 77 aA \\
\hline MGS GRAP084 & $35 \mathrm{bA}$ & 9 & $\mathrm{cB}$ & $38 \mathrm{cA}$ & 59 bA \\
\hline MGS NEBLINA & $11 \mathrm{cA}$ & 2 & $c A$ & $50 \mathrm{bA}$ & $36 \mathrm{cA}$ \\
\hline Cornicabra & $1 \mathrm{cA}$ & 3 & $c A$ & 64 bA & $16 \mathrm{~dB}$ \\
\hline Empeltre & $1 \mathrm{cA}$ & 0 & $c A$ & $47 \mathrm{cA}$ & $36 \mathrm{cA}$ \\
\hline Galega & $4 \mathrm{cA}$ & 0 & $c A$ & $85 \mathrm{aA}$ & $75 \mathrm{cA}$ \\
\hline MGS GRAP541 & $12 \mathrm{cA}$ & 7 & $c A$ & 56 bA & 52 bA \\
\hline MGS GRAP550 & $16 \mathrm{cA}$ & 11 & cA & $36 \mathrm{cA}$ & $41 \mathrm{cA}$ \\
\hline MGS GRAP553 & $1 \mathrm{cA}$ & 1 & cA & $29 \mathrm{cA}$ & $5 \mathrm{~dB}$ \\
\hline MGS GRAP556 & $9 \mathrm{cA}$ & 6 & cA & $50 \mathrm{bB}$ & 73 aA \\
\hline MGS GRAP561 & $6 \mathrm{cA}$ & 1 & $c A$ & $44 \mathrm{cB}$ & $72 \mathrm{aA}$ \\
\hline MGS GRAP575 & $6 \mathrm{cA}$ & 0 & $c A$ & $50 \mathrm{cA}$ & $50 \mathrm{cA}$ \\
\hline JB 1 & $1 \mathrm{cA}$ & 0 & CA & $63 \mathrm{bA}$ & 62 bA \\
\hline MGS MAN215 & 73 aA & 19 & bB & $15 \mathrm{~dB}$ & $39 \mathrm{cA}$ \\
\hline MGS MAN234 & $13 \mathrm{cA}$ & 19 & bA & 49 bA & $46 \mathrm{cA}$ \\
\hline MGS MARIENSE & $4 \mathrm{cA}$ & 0 & $c A$ & 74 aA & 62 bA \\
\hline Mission & $2 \mathrm{cA}$ & 0 & cA & 74 aA & 86 aA \\
\hline MGS MISS293 & $18 \mathrm{cA}$ & 13 & $c A$ & 65 bA & $41 \mathrm{cB}$ \\
\hline Negroa & $11 \mathrm{cA}$ & 0 & $c A$ & 59 bA & 75 aA \\
\hline Penafiel SP & $23 \mathrm{cA}$ & 0 & $\mathrm{cB}$ & $31 \mathrm{cB}$ & $63 \mathrm{bA}$ \\
\hline Picual & $17 \mathrm{cA}$ & 13 & cA & 56 bA & $68 \mathrm{aA}$ \\
\hline MGS ROP398 & CA & 2 & $c A$ & $34 \mathrm{cA}$ & $32 \mathrm{cA}$ \\
\hline MGS SAL488 & $6 \mathrm{cA}$ & 1 & $c A$ & $64 \mathrm{bB}$ & 86 aA \\
\hline Santa Catalina & 32 bA & 11 & $c B$ & $37 \mathrm{cB}$ & $60 \mathrm{bA}$ \\
\hline MGS TAF390 & 73 aA & 45 & $\mathrm{aB}$ & $9 \mathrm{~dB}$ & $44 \mathrm{cA}$ \\
\hline MGS TAF391 & $44 \mathrm{bA}$ & 19 & $b B$ & $32 \mathrm{cB}$ & 54 bA \\
\hline CV (\%) & & & & & \\
\hline
\end{tabular}

Médias seguidas de mesma letra minúscula nas colunas e maiúsculas nas linhas não diferem entre si pelo teste de Scott-Knott a 5\% de probabilidade de erro. fenológico em que ela está e, caso os níveis de carboidratos estejam adequados, há influência positiva no enraizamento. Com o fim da fase produtiva das plantas de oliveira em meados de fevereiro e março, em abril elas retomam seu crescimento e produção de assimilados, o que favorece o processo de enraizamento, pois as estacas possuem maior quantidade de substâncias de reserva. Já em agosto, com as temperaturas baixas, as plantas de oliveira diminuem seu metabolismo e as substâncias de reservas são direcionadas ao sistema radicular, diminuindo seu teor nas estacas, o que compromete o processo de enraizamento.

A formação de raízes adventícias em estacas pode ser direta e indiretamente controlada por genes, sendo a potencialidade de uma estaca em formar raízes variáveis com a espécie e cultivar. Pode ser feita uma classificação entre espécies ou cultivares de fácil, médio ou difícil capacidade em emitir raízes, ainda que a facilidade de enraizamento seja resultante da interaçáo de diversos fatores e não apenas do potencial genético (PIo et al., 2010). Caso a espécie ou cultivar tenha dificuldade em emitir raízes, pode ser observada apenas a formaçáo de calos, ou seja, um crescimento de células de forma desordenada responsável pela cicatrização do corte efetuado na base da estaca (Han et al., 2009).

Os percentuais de enraizamento atingidos por algumas cultivares, neste trabalho, superam o apontado por Oliveira et al. (2003), que registraram 20,8\% de enraizamento para estacas de oliveira da cultivar Ascolano 315 ('MGS ASC315') coletadas em abril, no Sul de Minas Gerais. A variação do potencial de enraizamento entre diferentes cultivares de oliveira, foi também verificada por DEL Río e CABALlero (2005), ao avaliarem 152 cultivares, com percentuais de enraizamento variando de 0 a $89 \%$.

$\mathrm{O}$ grupo com a maior porcentagem de estacas com calos foi obtido pelas cultivares MGS ASC323 (81\%), MGS VIGLIONE (82\%), Galega (85\%), MGS MARIENSE (74\%) e Mission (74\%), quando coletadas em abril (Tabela 1). Para a estaquia em agosto, maiores porcentagens foram registradas nas cultivares Alto D'Ouro (37\%), Clone 0075 (77\%), MGS GRAP556 (73\%), MGS GRAP561 (72\%), Mission (86\%), Negroa (75\%), Picual (68\%) e MGS SAL488 (86\%).

A formação de calo na base das estacas possui relação com a formação de raízes. Ambos os eventos ocorrem de maneira independente, porém necessitam das mesmas condiçôes para sua ocorrência (HaN et al., 2009). A presença de calos nas estacas indica diferenciaçōes celulares conhecidas como estruturas indicadoras da formação dos primórdios de raízes adventícias. Algumas cultivares de oliveira necessitam de um tempo maior para que ocorra esta diferenciação celular e, consequentemente, a formação de raízes adventícias após o processo de formação de calos.

Para o número médio de raízes, o grupo formado pelas cultivares Arbequina $(6,0)$, Ascolano $322(5,5)$, Cerignola (6,2), Clone $0010(4,8)$, Galega, (6,0), Grappolo $541(9,2)$, 
Grappolo 575 (7,1), Manzanilla 215 (6,5), Negroa (5,6), Penafiel SP $(8,1)$, Picual $(6,5)$, Santa Catalina $(6,0)$, Tafahi $390(9,6)$ e Tafahi $391(6,1)$ proporcionaram melhores resultados quando coletadas em abril. Em agosto, estacas da cultivar MGS GRAP541 (11,0) proporcionaram maiores valores (Tabela 2). No geral, observou-se grande variação entre a emissão de raízes nas estacas das diferentes cultivares. Porém, estacas das cultivares de oliveira coletadas em abril tiveram maior emissão de raízes. Os resultados registrados para a cultivar MGS ASC315 (Ascolano 315) corroboram os de Oliveira et al. (2003), que obtiveram 6,2 raízes em estacas coletadas em abril e os de Oliveira et al. (2009), que obtiveram 2,5 raízes em estacas coletadas em agosto.

Tabela 2. Número e comprimento médio de raízes em estacas semilenhosas de cultivares de oliveira, coletadas em diferentes épocas do ano (abril e agosto)

\begin{tabular}{|c|c|c|c|c|}
\hline \multirow{3}{*}{$\begin{array}{l}\text { Cultivar } \\
\text { Alto D'Ouro }\end{array}$} & \multicolumn{2}{|c|}{ Numero de raízes } & \multirow{2}{*}{\multicolumn{2}{|c|}{$\begin{array}{l}\text { Comprimento } \\
\text { de raízes }(\mathrm{cm})\end{array}$}} \\
\hline & abril & agosto & & \\
\hline & 2,2 bA & $0,0 \quad c A$ & 0,6 & c \\
\hline Arbequina & 6,0 aA & $2,7 \quad c A$ & 2,0 & $b$ \\
\hline MGS ASC315 & 7,7 aA & $2,4 \quad c A$ & 2,0 & $b$ \\
\hline MGS ASC 322 & 5,5 aA & $4,6 \quad b A$ & 3,7 & $\mathrm{a}$ \\
\hline MGS ASC 323 & 3,8 bA & $0,0 \quad c B$ & 1,6 & $b$ \\
\hline Ascolano USA & $4,0 \quad b A$ & 3,3 bA & 2,9 & a \\
\hline Cerignola & $6,2 \mathrm{aA}$ & 3,3 bA & 2,0 & $b$ \\
\hline MGS ZAL010 & 4,8 aA & 3,4 bA & 1,9 & $b$ \\
\hline MGS SEVERO & 2,2 bA & 5,6 bA & 2,3 & $b$ \\
\hline MGS VIVLIONE & $1,0 \quad b A$ & $0,0 \quad c A$ & 1,0 & c \\
\hline MGS GRAP084 & 3,0 bA & $2,8 \quad c A$ & 2,6 & $\mathrm{a}$ \\
\hline MGS NEBLINA & 2,8 bA & $1,6 \mathrm{cA}$ & 1,2 & c \\
\hline Cornicabra & 0,5 bA & $0,4 \quad c A$ & 0,3 & c \\
\hline Empeltre & 1,0 bA & $0,0 \quad c A$ & 0,2 & c \\
\hline Galega & 6,0 aA & $0,0 \quad c B$ & 0,5 & c \\
\hline MGS GRAP541 & 9,2 aA & 11,0 aA & 0,6 & c \\
\hline MGS GRAP550 & 3,7 bA & $2,7 \quad c A$ & 1,6 & b \\
\hline MGS GRAP553 & $2,0 \quad b A$ & $0,3 \mathrm{cA}$ & 0,4 & c \\
\hline MGS GRAP556 & 2,2 bA & $2,5 \mathrm{cA}$ & 2,6 & $\mathrm{a}$ \\
\hline MGS GRAP561 & 3,0 bA & $1,8 \mathrm{cA}$ & 0,6 & c \\
\hline MGS GRAP575 & 7,1 aA & $0,0 \quad c B$ & 0,8 & c \\
\hline JB 1 & 0,8 bA & $0,0 \quad c A$ & 0,1 & c \\
\hline MGS MAN215 & 6,5 aA & $2,5 \quad \mathrm{cB}$ & 2,2 & $b$ \\
\hline MGS MAN234 & $1,9 \mathrm{bA}$ & $4,8 \quad b A$ & 2,2 & $b$ \\
\hline MGS MARIENSE & $2,1 \quad b A$ & $0,0 \quad c A$ & 0,8 & c \\
\hline Mission & 0,5 bA & $0,0 \quad c A$ & 0,6 & c \\
\hline MGS MISS293 & 4,4 bA & 3,3 bA & 3,2 & a \\
\hline Negroa & 5,6 aA & $0,0 \quad c B$ & 0,9 & c \\
\hline Penafiel SP & 8,1 aA & $0,0 \quad c B$ & 1,4 & b \\
\hline Picual & 6,5 aA & 3,7 bA & 2,6 & a \\
\hline MGS ROP398 & 4,2 bA & $0,5 \mathrm{cB}$ & 0,6 & c \\
\hline MGS SAL488 & $1,7 \quad b A$ & $0,3 \quad c A$ & 0,8 & c \\
\hline Santa Catalina & 6,0 aA & $1,9 \quad \mathrm{cB}$ & 1,2 & c \\
\hline MGS TAF390 & 9,6 aA & 6,7 bA & 3,0 & $\mathrm{a}$ \\
\hline MGS TAF391 & 6,1 aA & $2,1 \quad c B$ & 2,0 & $b$ \\
\hline C.V. (\%) & & & 32 & \\
\hline
\end{tabular}

Médias seguidas de mesma letra minúscula nas colunas e maiúsculas nas linhas nấo diferem entre si pelo teste de Scott-Knott a 5\% de probabilidade de erro.
Maiores comprimentos de raízes por estaca foram observados nas cultivares MGS ASC322 (3,7 $\mathrm{cm})$, Ascolano USA $(2,9 \mathrm{~cm})$, MGS GRAP084, MGS GRAP556 e Picual $(2,6 \mathrm{~cm})$, MGS MISS293 $(3,2 \mathrm{~cm})$ e MGS TAF390 $(3,0 \mathrm{~cm})$ (Tabela 2). A estaquia em abril propiciou maior comprimento médio de raízes $(2,1 \mathrm{~cm})$, em relação à estaquia em agosto $(1,0 \mathrm{~cm})$ para as cultivares estudadas. Essa diferença entre o comprimento das estacas em relaçáo às épocas em que foram coletadas pode estar relacionada ao estádio fenológico, o que pode ocorrer alteração no conteúdo de cofatores e acúmulo de inibidores do enraizamento, que nesse caso pode influenciar ou não na elongação das raízes (HAN et al., 2009).

Concluindo, o potencial de enraizamento das estacas varia entre as cultivares de oliveira. As cultivares MGS MANZ215 e MGS TAF390 se destacaram pela maior capacidade de enraizamento. A estaquia em abril favorece o enraizamento, em relação à estaquia realizada em agosto, para algumas das cultivares avaliadas.

\section{AGRADECIMENTOS}

À Fundação de Amparo a Pesquisa do Estado de Minas Gerais - FAPEMIG (CAG APQ 02721/09) pelo apoio financeiro na execução desse trabalho e à Coordenação de Aperfeiçoamento de Pessoal de Nível Superior (CAPES), pela concessáo de bolsa de mestrado ao primeiro autor.

\section{REFERÊNCIAS}

BRASIL. Projeto de Lei de 2012. Disponível em: <http://www. camara.gov.br/proposicoesWeb/prop_mostrarintegra;jsessionid $=15$ 04CCC0687D763D8C1F06CA23003F74.node1? codteor=96569 0\&filename $=P L+3260 / 2012>$. Acesso em: 24/4/2012.

DANELUZ, S.; PIO, R.; CHAGAS, E.A.; BARBOSA, W.; OHLAND, T.; KOTZ, T.E. Propagaçấo da figueira 'Roxo de Valinhos' por alporquia. Revista Brasileira de Fruticultura, v.31, p.285-290, 2009.

DEL RÍO, C.; CABALLERO, J.M. Aptitut al enrazaizamento. In: RALLO, L.; BARRANCO, D.; CABALLERO, J.M.; DEL RÍO, C.; MARTÍN, A.; TOUS, J.; TRUJILLO, I. (Ed.). Variedades de olivo en España. Sevilla: Consejería de Agricultura y Pesca de la Junta de Andalucía/Mundi-Prensa, 2005. Cap.4, p.277-280.

FERREIRA, D.F. Sisvar: um programa para análises e ensino de estatística. Revista Symposium, v.6, p.36-41, 2008.

HAN, H.; ZHANG, S.; SUN, X. A review on the molecular mechanism of plants rooting modulated by auxin. African Journal of Biotechnology, v.8, p.348-353, 2009.

OHLAND, T.; PIO, R.; CHAGAS, E.A.; BARBOSA, W.; KOTZ, T.E.; DANELUZ, S. Enraizamento de estacas apicais de figueira 'Roxo de Valinhos' em funçáo de época de coleta e AIB. Ciência e Agrotecnologia, v.33, p.74-78, 2009. 
OLIVEIRA, A.F.; PASQUAL, M.; CHALFUN, N.N.J.; REGINA, M.A.; RINCÓN, C.D.R. Enraizamento de estacas semilenhosas de oliveira sob diferentes épocas, substratos e concentraçóes de ácido indolbutírico. Ciência e Agrotecnologia, v.27, p.117-125, 2003.

OLIVEIRA, A.F.; CHALFUN, N.N.J.; ALVARENGA, A.A.; VIEIRA NETO, J.; PIO, R.; OLIVEIRA, D.L. Estaquia de oliveira em diferentes épocas, substratos e doses de AIB diluído em $\mathrm{NaOH}$ e álcool. Ciência e Agrotecnologia, v.33, p.79-85, 2009.

OLIVEIRA, M.C.; RAMOS, J.D.; PIO, R.; CARDOSO, M.G. Características fenológicas e físicas e perfil de ácidos graxos em oliveiras no sul de Minas Gerais. Pesquisa Agropecuária Brasileira, v.47, p.30-35, 2012.

OLIVEIRA, M.C.; VIEIRA NETO, J.; OLIVEIRA, R.S.; PIO, R.; OLIVEIRA, N.C.; RAMOS, J.D. Enraizamento de estacas de duas cultivares de oliveira submetidas à aplicaçáo de diferentes fertilizantes. Bragantia, v.69, p.99-103, 2010a.

OLIVEIRA, M.C.; VIEIRA NETO, J.; PIO, R.; OLIVEIRA, A.F.; RAMOS, J.D. Enraizamento de estacas de oliveira submetidas a aplicação de fertilizantes orgânicos e AIB. Ciência e Agrotecnologia, v.34, p.337-344, 2010 b.

PIO, R.; BASTOS, D.C.; BERTI, A.J.; SCARPARE FILHO, J.A.; MOURÃO FILHO, F.A.A.; ENTELMANN, F.A.; ALVES,
A.S.R.; BETTIOL NETO, J.E. Enraizamento de diferentes tipos de estacas de oliveira (Olea europaea L.) utilizando ácido indolbutírico. Ciência e Agrotecnologia, v.29, p.562-567, 2005.

PIO, R.; CAMPO DALL'ORTO, F.A.; ALVARENGA, A.A.; ABRAHÃO, E.; CHAGAS, E.A.; SIGNORINI, G. Propagação do marmeleiro 'Japonês' por estaquia e alporquia realizadas em diferentes épocas. Ciência e Agrotecnologia, v.31, p.570-574, 2007.

PIO, R.; COSTA, F.C.; CURI, P.N.; MOURA, P.H.M. Enraizamento de estacas lenhosas de cultivares de kiwizeiro. Scientia Agraria, v.11, p.271-274, 2010.

SILVA, L.F.O.; OLIVEIRA, A.F.; PIO, R.; ALVES, T.C.; ZAMBON, C.R. Variaçáo na qualidade do azeite em cultivares de oliveira. Bragantia, v.71, p.202-209, 2012.

SMARSI, R.C.; CHAGAS, E.A.; REIS, L.L.; OLIVEIRA, G.F.; MENDONÇA, V.; TROPALDI, L.; PIO, R.; SCARPARE FILHO, J.A. Concentraçóes de ácido indolbutírico e tipos de substrato na propagaçáo vegetativa de lichia. Revista Brasileira de Fruticultura, v.30, p.7-11, 2008.

VÍLLA, F; PIO, R.; CHALFUN, N.N.J.; GONTIJO, T.C.A.; COELHO, J.H.C.; DUTRA, L.F. Enraizamento de estacas herbáceas do porta-enxerto de videira 'Riparia de traviú' tratadas com auxinas. Ciência e Agrotecnologia, v.27, p.1426-1431, 2003. 